\title{
MULTIFRACTAL ANALYSIS OF WEAK GIBBS MEASURES FOR NON-UNIFORMLY EXPANDING $C^{1}$ MAPS
}

\author{
THOMAS JORDAN AND MICHAE RAMS
}

\begin{abstract}
We will consider the local dimension spectrum of a weak Gibbs measure on a $C^{1}$ non-uniformly hyperbolic system of Manneville-Pomeau type. We will present the spectrum in three ways: using invariant measures, ergodic invariant measures supported on hyperbolic sets and equilibrium states. We are also proving analyticity of the spectrum under additional assumptions. All three presentations are well known for smooth uniformly hyperbolic systems.
\end{abstract}

The theory of multifractal analysis for hyperbolic conformal dynamical systems is now extremely well developed. There are complete results for local dimension of Gibbs measures, Lyapunov spectra and Birkhoff spectra. For early results on the local dimension spectra see [3] and [18], for a general description see [16] and for more specific and very general results see [1] and [15]. However, the picture is not complete for non-uniformly hyperbolic systems. There are results for specific nonuniformly expanding systems by Kesseböhmer and Stratmann ([11] and [12]) and in the case of complex dynamics by Byrne, [2] .

As our interest is in the local dimension spectrum for invariant measures, five papers are of special interest for us. The first results about local dimension were in the papers by Nakaishi and by Pollicott and Weiss, [14, [17]. They obtained the local dimension spectrum for the measure of maximal entropy for a restricted class of parabolic maps without critical points. Stratmann and Urbański in [19] and [20] considered complex (hence analytic) maps with possible critical points. However, the analyticity assumption in these papers could be probably reduced to assumptions from section 3 of [7]. In Theorem 4 of [24] Yuri

\footnotetext{
We would like to thank the referee of this paper for their detailed comments which certainly helped improve the paper. The research of M.R. was supported by grants EU FP6 ToK SPADE2, EU FP6 RTN CODY and MNiSW grant 'Chaos, fraktale i dynamika konforemna'. The research was started during a visit of M. R. to Bristol. M. R. would like to thank Bristol University for the hospitality shown during his visit.
} 
computes a portion of the multifractal spectra for weak Gibbs measures of some non-uniformly hyperbolic systems.

The aim of this paper is to obtain a complete spectrum for the local dimension of weak Gibbs measures for $C^{1}$ non-uniformly hyperbolic systems. We will include results about the points where the local dimension is infinite, a phenomenon which does not occur in the uniformly hyperbolic setting. We will be considering systems with parabolic periodic points but no critical points. Well known examples of such maps include the Manneville-Pomeau map and the Farey map.

The methods we use are adapted from the papers [6] and [9] where the Lyapunov and Birkhoff spectra of such maps are considered. For most of the paper we work directly with the original system, without inducing, which lets us omit the usual assumptions about behaviour of the map around parabolic points (except for Theorem 4 which deals with analyticity of the spectra).

\section{Notation AND RESUlts}

We consider non-uniformly expanding one-dimensional Markov maps. More precisely, let $I=[0,1]$. Let $\left\{I_{i}\right\}, i=1, \ldots, p$ be closed subintervals of $I$ with disjoint interiors. Let $A$ be a $p \times p$ matrix consisting of 0 's and 1's where there exists $k \in \mathbb{N}$ such that for all $i, j A^{k+1}(i, j)>0$. Let $T_{i}: I \rightarrow \mathbb{R}$ be $C^{1}$ bijective diffeomorphisms with closed domains $J_{i} \subset I_{i}$ for which $I_{j} \subset T_{i}\left(J_{i}\right)$ if $A(i, j)=1$ and $T_{i}\left(J_{i}\right) \cap \operatorname{int} I_{j}=\emptyset$ if $A(i, j)=0$. We will let $\Lambda_{0}=\cup_{i} J_{i}$ and let $T: \Lambda_{0} \rightarrow I$ be defined as $T_{i}$ on $J_{i}$. When $J_{i} \cap J_{j}=\{x\}$ and $i<j$ we will take $T(x)=T_{i}(x)$. We will assume that $\left|T_{i}^{\prime}(x)\right| \geq 1$ at every point $x \in J_{i}$ and that there are at most countably many points with derivative \pm 1 . We will denote by $\Lambda$ the set of points whose trajectory never leaves $\Lambda_{0}$.

We will allow the existence of parabolic periodic orbits $\left\{x, T(x), \ldots, T^{m-1}(x), T^{m}(x)=x\right\}$ where the derivative of $T$ will be \pm 1 at all points on the orbit. The existence of a parabolic orbit implies that the map is non-uniformly expanding.

Let $\Sigma=\{1, \ldots, p\}^{\mathbb{N}}$ be the full shiftspace with the usual left shift $\sigma$ and $\Sigma_{A} \subset \Sigma$ the subshift with respect to the matrix $A$. For $\underline{i} \in \Sigma$ we will denote by $i_{n}$ its $n$-th element and by $i^{n}$ the sequence of its first $n$ elements. For some $0<\beta<1$ we will use the metric $d_{\kappa}$ on $\Sigma_{A}$ given by $d_{\kappa}(\underline{i}, \underline{j})=\kappa^{|\underline{i} \wedge \underline{j}|}$ where $|\underline{i} \wedge \underline{j}|=\inf \left\{m \in \mathbb{N}: i_{m} \neq j_{m}\right\}-1$. By our assumptions, $\left(\Sigma_{A}, \sigma\right)$ is topologically transitive. Let $\Pi: \Sigma_{A} \rightarrow \Lambda$ be defined by

$$
\Pi(\underline{i})=\lim _{n \rightarrow \infty} T_{i_{1}}^{-1} \circ \cdots \circ T_{i_{n}}^{-1}(\Lambda)
$$


(the limit is always one point, see the proof of Lemma 2.1 in [21]). The local dimension of a measure $\mu$ at a point $x$ is defined by

$$
d_{\mu}(x)=\lim _{r \rightarrow 0} \frac{\log \mu(B(x, r))}{\log r}
$$

when this limit exists.

Let $\phi: \Sigma_{A} \rightarrow \mathbb{R}$ be a continuous potential. We call a $\sigma$-invariant probabilistic measure $\tilde{\nu}$ supported on $\Sigma_{A}$ weak Gibbs for the potential $\phi$ if there exists a constant $P$ and a decreasing sequence $\left\{k_{n}\right\}$ such that $\lim _{n \rightarrow \infty} k_{n}=0$ and for every $\underline{i} \in \Sigma_{A}$ and for every $n$

$$
\exp \left(-n k_{n}\right) \leq \frac{\tilde{\nu}\left(\left[i_{1} \ldots i_{n}\right]\right)}{\exp \left(S_{n} \phi(\underline{i})-n P\right)} \leq \exp \left(n k_{n}\right) .
$$

This is similar to the definition in 23 except we require the result to hold for all sequences not just for $\tilde{\nu}$-almost all sequences. The existence of such weak Gibbs measures for all continuous potentials is established in $[10$.

For the rest of the paper let $\tilde{\nu}$ be the weak Gibbs measure for the potential $\phi$ and $\nu=\tilde{\nu} \circ \Pi^{-1}$. Our goal will be to describe the local dimension spectrum function $\alpha \rightarrow \operatorname{dim}_{H} X_{\alpha}$, where

$$
X_{\alpha}=\left\{x \in \Lambda: d_{\nu}(x)=\alpha\right\} .
$$

Since $\tilde{\nu}$ is invariant under the map $\sigma$ it follows that $\nu$ is invariant under $T$. We will assume that $P(\phi)=0$ and $\phi(x)<0$ at each $x \in \Sigma_{A}$. Note here that this assumption is not very restrictive in the narrower class of Hölder potentials. If $\phi$ is Hölder continuous and does not satisfy this assumption then it is possible to add a constant and a coboundary to obtain a new potential which will satisfy these conditions (see Theorem 9 in [4] and note that Gibbs measures for Hölder potentials on subshifts of finite type have positive entropy).

Let us introduce the following notation, let $\mathcal{M}_{T}(\Lambda)$ be the set of T-invariant probability measures on $\Lambda$ and $\mathcal{M}_{\sigma^{n}}\left(\Sigma_{A}\right)$ be the set of $\sigma^{n}$ invariant probability measures on $\Sigma_{A}$. For $\mu \in M_{\sigma}\left(\Sigma_{A}\right)$ let $h\left(\mu, \sigma^{n}\right)$ denote the entropy of $\mu$ with respect to $\sigma^{n}$ (recall that by Abramov's Theorem $n h(\mu, \sigma)=h\left(\mu, \sigma^{n}\right)$ for $\left.\mu \in \mathcal{M}_{\sigma}\left(\Sigma_{A}\right)\right)$. Let $\psi: \Sigma_{A} \rightarrow \mathbb{R}$ be defined by $\psi(\underline{i})=\log \left|T_{i_{1}}^{\prime}(\Pi(\underline{i}))\right|$ and for $\mu \in M_{\sigma}\left(\Sigma_{A}\right)$ we define the Lyapunov exponent of the measure $\mu$ as

$$
\lambda(\mu)=\int \psi(\underline{i}) \mathrm{d} \mu(\underline{i}) .
$$

We will call an ergodic measure $\mu \circ \Pi^{-1}$ hyperbolic if $\lambda(\mu)>0$. We will use a more restricted family of measures as well. An ergodic measure for which the support does not contain any parabolic periodic orbits will 
be called uniformly hyperbolic. Moreover we will denote the family of uniformly hyperbolic measures by $\mathcal{M}_{H}\left(\Sigma_{A}\right)$. Obviously, any uniformly hyperbolic measure is hyperbolic. Similarly, closed and $T$-forward invariant subsets of $\Lambda$ that do not contain any parabolic periodic orbits will be called hyperbolic sets and the family of all hyperbolic sets will be denoted by $\mathcal{H}$. The family of hyperbolic sets on which $T$ is an uniformly expanding subshift of finite type will be denoted by $\mathcal{H}_{0}$. Let

$$
\alpha_{\min }=\min _{\mu \in \mathcal{M}_{\sigma}\left(\Sigma_{A}\right)}\left\{-\frac{\int \phi \mathrm{d} \mu}{\lambda(\mu)}: \lambda(\mu)>0\right\}
$$

and

$$
\alpha_{\max }=\max _{\mu \in \mathcal{M}_{\sigma}\left(\Sigma_{A}\right)}\left\{-\frac{\int \phi \mathrm{d} \mu}{\lambda(\mu)}: \lambda(\mu)>0\right\} .
$$

Clearly, $\alpha_{\max }=\infty$ if and only if $\Lambda$ contains a parabolic periodic orbit (if: consider linear combinations of a measure with positive Lyapunov exponent and the invariant measure supported on the parabolic periodic orbit; only if: $-\frac{1}{n} S_{n} \phi$ is always bounded and $\frac{1}{n} S_{n} \psi$ is bounded away from zero in the absence of parabolic points).

We can now state our main results.

Theorem 1. Let $(X, T)$ be non-uniformly expanding one-dimensional Markov map and let $\mu$ be a weak Gibbs measure for the potential $\phi$. Then

1. $X_{\alpha}$ is empty for $\alpha<\alpha_{\min }$ or $\alpha>\alpha_{\max }$.

2. The function $\alpha \rightarrow \operatorname{dim}_{H} X_{\alpha}$ is continuous on $\left(\alpha_{\min }, \alpha_{\max }\right)$ and right-continuous at $\alpha_{\min }$. If $\alpha_{\max }<\infty$ then it is also leftcontinuous at $\alpha_{\max }$.

3. For any $\alpha \in\left[\alpha_{\min }, \alpha_{\max }\right] \backslash\{\infty\}$ we have that

$$
\operatorname{dim}_{H} X_{\alpha}=\sup _{\mu \in \mathcal{M}_{\sigma}\left(\Sigma_{A}\right)}\left\{\frac{h(\mu, \sigma)}{\lambda(\mu)}:-\frac{\int \phi d \mu}{\lambda(\mu)}=\alpha \text { and } \lambda(\mu)>0\right\} \text {. }
$$

4. If $\alpha_{\max }=\infty$ then $\operatorname{dim}_{H} X_{\alpha}$ is a nondecreasing function of $\alpha$ and

$$
\operatorname{dim}_{H} X_{\infty}=\operatorname{dim}_{H} \Lambda \text {. }
$$

In addition $\operatorname{dim}_{H} X_{\alpha}$ can be described as a supremum over the dimension of $X_{\alpha}$ restricted to hyperbolic subsets.

Theorem 2. Under assumptions of Theorem 1 , for any $\alpha \in\left(\alpha_{\min }, \alpha_{\max }\right)$ we have that

$$
\operatorname{dim}_{H} X_{\alpha}=\sup _{B \in \mathcal{H}_{0}} \operatorname{dim}_{H}\left(B \cap X_{\alpha}\right)=\sup _{\mu \in \mathcal{M}_{H}\left(\Sigma_{A}\right)}\left\{\frac{h(\mu, \sigma)}{\lambda(\mu)}:-\frac{\int \phi d \mu}{\lambda(\mu)}=\alpha\right\} .
$$


Consider the family of potentials $\psi_{a}=a \psi+b(a) \phi$, where $b(a)$ is the unique number for which $P\left(\psi_{a}\right)=0$. Like in the hyperbolic case, the local dimension spectrum can be written as the Lagrange-Fenchel transform of the function $b$.

Theorem 3. Under the assumptions of Theorem 1, for every $\alpha \in$ $\left(\alpha_{\min }, \alpha_{\max }\right)$ we have that

(1) $\operatorname{dim}_{H} X_{\alpha}=\inf _{a}\{b(a) \alpha-a\}$.

(2) There exists $a \in \mathbb{R}$ and $\mu_{a} \in M_{\sigma}\left(\Sigma_{A}\right)$ such that $\mu_{a}$ is an equilibrium state for $\psi_{a},-\int \phi d \mu_{a} / \lambda\left(\mu_{a}\right)=\alpha$ and

$$
\operatorname{dim}_{H} X_{\alpha}=\frac{h\left(\mu_{\alpha}, \sigma\right)}{\lambda\left(\mu_{\alpha}\right)}
$$

(3) The function $\alpha \rightarrow \operatorname{dim}_{H} X_{\alpha}$ is concave on $\left[\alpha_{\min }, \alpha_{\max }\right] \backslash\{\infty\}$.

Let us add some remarks, comparing our results for parabolic maps with the usual behaviour of expanding maps. Since the space of invariant measures is closed under the weak* topology and the entropy is upper semi-continuous, the supremum in Theorem 1 is always achieved. However, in contrast to the uniformly hyperbolic situation, this is not always the case with Theorem 2. Indeed, consider a MannevillePomeau map for which an absolutely continuous, invariant, probability measure $\nu_{0}$ exists. Let $\phi \equiv-\log 2$. In this situation the Hausdorff dimension of $X_{\alpha}$ equals the Hausdorff dimension of the set of points for which the Lyapunov exponent equals $\log 2 / \alpha$ (compare Theorem 2 with [6]). If we let $\alpha>\alpha_{0}=\log 2 / \lambda\left(\nu_{0}\right)$ then we can deduce from the results in [6] that $\operatorname{dim}_{H} X_{\alpha}=1$. However, there cannot exist any hyperbolic sets of dimension 1 (see [5] ), thus neither of the suprema in Theorem 2 are achieved.

Moreover, note that the supremum in Theorem 1 is not necessarily achieved among ergodic measures (i.e. dropping the uniform hyperbolicity assumption from Theorem [2). Indeed, in the example above $\nu_{0}$ is the unique ergodic measure of dimension 1 ([13]) and hence for $\alpha>\alpha_{0}$ the supremum in Theorem 1 cannot be obtained by an ergodic measure. This is not a contradiction with Theorem 3 for the following reason. For all $a \leq-1$, corresponding to $\alpha \geq \alpha_{0}$, we have $b(a)=0$ and the Dirac measure at the parabolic point $\delta_{0}$ is an ergodic equilibrium state. For $a=-1$ we have another ergodic equilibrium state, the absolutely continuous measure $\nu_{0} \mu_{a}=\nu_{0}$, and all the linear combinations of $\delta_{0}$ and $\nu_{0}$ will be (non-ergodic) equilibrium states too. For all $\alpha>\alpha_{0}$ the equilibrium state provided by Theorem 2 is one of those linear combinations, hence it is invariant but not ergodic. 
For all the above results we only need the tempered distortion property, which easily follows from our assumptions (see Lemma 1). However, to prove the last result of our paper we need something more. We refer the reader to the second section of [21] where these additional assumptions were first introduced, we only want to mention here that whilst one can construct examples when they are not satisfied, they hold for all the most important examples like the Manneville-Pomeau map and the Farey map.

Theorem 4. Assume that $T$ is $C^{1+\theta}$ and our potential $\phi$ is $C^{\theta}$ for some $\theta>0$. We also assume that for every parabolic point $\omega$ of period $n\left|\left(T^{n}\right)^{\prime}\right|$ is monotone on sufficiently small one-sided neighbourhoods of $\omega$ and that there exist constants $L>0$ and $0<\beta<\frac{\theta}{(1-\theta)}$ (or $\beta \in \mathbb{R}_{+}$ if $T$ is $C^{2}$ ) such that

$$
L^{-1} \leq \liminf _{x \rightarrow \omega} \frac{||\left(T^{n}\right)^{\prime}(x)|-1|}{|x-\omega|^{\beta}} \leq \limsup _{x \rightarrow \omega} \frac{||\left(T^{n}\right)^{\prime}(x)|-1|}{|x-\omega|^{\beta}} \leq L
$$

Then the function $\alpha \rightarrow \operatorname{dim}_{H} X_{\alpha}$ is real analytic on $\left(\alpha_{\min }, \alpha_{\max }\right)$ with exception of at most one point.

More precisely, this function is real analytic if and only if no SRB measure exists, otherwise it is constant (equal to $\operatorname{dim}_{H} \Lambda$ ) for sufficiently big $\alpha$. We comment a bit more on this at the end of section 7 .

Let us remark here that for the measure of maximal entropy its level set for local dimension $\alpha$ coincides with the level set for Lyapunov exponent $\frac{h}{\alpha}$. Hence, the piecewise analyticity of the local dimension spectrum for the measure of maximal entropy implies piecewise analyticity of the Lyapunov spectrum. By applying the results from [6] this implies the piecewise analyticity of $P\left(t \log \left|T^{\prime}\right|\right)$ (the only place where the analyticity will fail is at the solution to $P\left(t \log \left|T^{\prime}\right|\right)=0$.)

The paper is structured as follows. In section 2 we prove some preparatory lemmas relating the local dimension to the symbolic dynamics. Section 3 deals with the question of relating upper bounds for $X_{\alpha}$ to invariant measures which is a key ingredient for the upper bound in part 3 of Theorem 1 and the upper bound in Theorem 2. Section 4 contains the proof for Theorem 1, Theorems 2,3,4, are then proved in sections 5,6 and 7 respectively.

\section{Symbolic DYNAMiCS AND LOCAL DIMENSION}

A key technique in the proof of Theorem 1 will be to relate cylinder sets in the shift space to sets in $I$. We will denote

$$
\Delta_{i_{1}, \ldots, i_{n}}=\operatorname{span} \Pi\left(\left[i_{1}, \ldots, i_{n}\right]\right) .
$$


We will also use the notation $\Delta_{n}(\underline{i})=\Delta_{i_{1} \ldots i_{n}}$. We will define $D_{n}$ : $\Sigma_{A} \rightarrow \mathbb{R}$ by

$$
D_{n}(\underline{i})=\operatorname{diam}\left(\Delta_{n}(\underline{i})\right) .
$$

The tempered distortion property guarantees that in most cases $\frac{1}{n} \log D_{n}(\underline{i})$ is close to $\frac{1}{n} S_{n} \psi(\underline{i})$.

Definition 1. We say that the tempered distortion holds for a potential $f: \Sigma_{A} \rightarrow \mathbb{R}$ if there exists a decreasing sequence $K_{n}(f)$ such that $\lim _{n \rightarrow \infty} K_{n}(f)=0$ and for any $\underline{i} \in \Sigma_{A}$ we have

$$
\sup \left\{S_{n} f(\underline{i})-S_{n} f(\underline{j}): \underline{i}, \underline{j} \in\left[i_{1}, \ldots, i_{n}\right]\right\} \leq n K_{n}(f) .
$$

It is clear that by compactedness any continuous potential on $\Sigma_{A}$ will satisfy this property. The following important property (which allowed us, among other things, to put a symbolic coding on $\Lambda$, as defined in the previous section) is the statement of Lemma 2.1 in [21].

$$
\lim _{n \rightarrow \infty} \max _{\underline{i} \in \Sigma_{A}} D_{n}(\underline{i})=0 .
$$

This Lemma has some additional assumptions however the proof of the Lemma clearly still works in the current setting. The following lemma is an immediate consequence.

Lemma 1. For any sequence $\underline{i} \in \Sigma_{A}$ the distortion of the map $T^{n}$ restricted to $\Delta_{n}(\underline{i})$ is bounded by $e^{n K_{n}(\psi)}$, independently of $\underline{i}$. In particular,

$$
e^{-n K_{n}(\psi)} \leq D_{n}(\underline{i}) e^{S_{n} \psi(\underline{i})} \leq e^{n K_{n}(\psi)} .
$$

We will denote

$$
\rho_{n}=\max \left(k_{n}, K_{n}(\phi), K_{n}(\psi)\right) .
$$

We now consider how close $x$ can be to the endpoints of cylinders. For a sequence $\underline{i} \in \Sigma_{A}$ we will define by $Z_{n}(\underline{i})$ the distance between $\Pi(\underline{i})$ and the endpoints of the $n$th level cylinder it lies in, i.e.

$$
Z_{n}(\underline{i})=d\left(\Pi(\underline{i}), \partial\left(\Delta_{n}(\underline{i})\right)\right) .
$$

Let

$$
E=\left\{\underline{i} \in \Sigma: \exists N \in \mathbb{N} \text { such that for } n \geq N Z_{n}(\underline{i})=0\right\}
$$

and note that this is a countable set which contains all the points where the map $\Pi$ is not bijective.

Lemma 2. There exists $K>0$ such that for any $\underline{i} \in \Sigma_{A} \backslash E$ for infinitely many $n \in N$

$$
\frac{Z_{n}(\underline{i})}{D_{n}(\underline{i})} e^{n \rho_{n}} \geq K
$$


Proof. Let $j_{1} j_{2}$ and $j_{3} j_{4}$ be the symbolic expansions of the leftmost and rightmost second level cylinders in $I$. We note that for $\underline{i} \notin E$ there will be infinitely many $n$ for which $\sigma^{n} \underline{i}$ will start with a sequence other than $j_{1} j_{2}$ or $j_{3} j_{4}$, that is,

$$
\Pi(\underline{i}) \in \Delta_{i_{1}, \ldots, i_{n}} \backslash\left(\Delta_{i_{1}, \ldots, i_{n} j_{1} j_{2}} \cup \Delta_{i_{1}, \ldots, i_{n} j_{3} j_{4}}\right)
$$

The triple $\left(\Delta_{i_{1}, \ldots, i_{n}}, \Delta_{i_{1}, \ldots, i_{n} j_{1} j_{2}}, \Delta_{i_{1}, \ldots, i_{n} j_{3} j_{4}}\right)$ gets mapped onto $\left(\Delta_{\emptyset}, \Delta_{j_{1} j_{2}}, \Delta_{j_{3} j_{4}}\right)$ by the map $T^{n}$. The result then follows from Lemma 1.

We now show that for typical points with respect to an ergodic measure with positive entropy we have an even stronger result.

Lemma 3. Let $\mu \in \mathcal{M}_{\sigma}\left(\Sigma_{A}\right)$ be ergodic and satisfy $h(\mu, \sigma)>0$. We have that for $\mu$ almost all $\underline{i}$

$$
\lim _{n \rightarrow \infty} \frac{1}{n} \log \left(\frac{Z_{n}(\underline{i})}{D_{n}(\underline{i})}\right)=0 .
$$

Proof. Fix such an ergodic measure $\mu$. For $\underline{i} \in \Sigma_{A}$ let

$$
k_{n}(\underline{i})=\max _{k}\left\{i_{n+1}=i_{n}, \ldots, i_{n+k}=i_{n}\right\} .
$$

Define $f: \Sigma_{A} \rightarrow \mathbb{R}^{p}$ by $f(\underline{i})=e_{i_{1}}$ (the $i_{1}$ th unit vector in $\mathbb{R}^{p}$ ) and note that by the Birkhoff Ergodic Theorem for $\mu$ almost all $\underline{i}$

$$
\lim _{n \rightarrow \infty} \frac{S_{n} f(\underline{i})}{n}=\int f \mathrm{~d} \mu
$$

and since $h(\mu)>0$ we have $\int f \mathrm{~d} \mu \in[0,1)^{p}$. However,

$$
S_{n+k_{n}(\underline{i})} f(\underline{i})=S_{n} f(\underline{i})+k_{n}(\underline{i}) e_{i_{n}} .
$$

Thus, for the limit in (2.1) to exist, we must have $k_{n}(\underline{i})=o(n)$. The result now follows by tempered distortion and the fact that (because of boundedness of $\left.\left|T^{\prime}\right|\right)$

$$
\max _{\underline{i} \in \Sigma_{A}}-\log D_{n}(\underline{i})=O(n) .
$$

\section{Covers of $X_{\alpha}$ And invariant measures}

In this section we relate covers of the sets $X_{\alpha}$ to certain invariant measures. This will allow us to show that

$$
\operatorname{dim}_{H} X_{\alpha} \leq \sup _{\mu \in \mathcal{M}\left(\Sigma_{A}\right)}\left\{\frac{h(\mu, \sigma)}{\lambda(\mu)}:-\frac{\int \phi \mathrm{d} \mu}{\lambda(\mu)}=\alpha\right\}
$$

which gives the upper bound for part 3 of Theorem 11. These results will also be needed to prove Theorem 2 . We start by giving a simple result relating $\sigma^{n}$ invariant measures to $\sigma$-invariant measures. 
Lemma 4. Let $\mu \in \mathcal{M}_{\sigma^{n}}\left(\Sigma_{A}\right)$. We can find an invariant measure $m \in \mathcal{M}_{\sigma}\left(\Sigma_{A}\right)$ such that

(1) $h(m, \sigma)=\frac{h\left(\mu, \sigma^{n}\right)}{n}$

(2) $\lambda(m, \sigma)=\frac{\int S_{n} \psi d \mu}{n}$

(3) $\int \phi d m=\frac{\int S_{n} \phi d \mu}{n}$

and if $\mu$ is ergodic with respect to $\sigma^{n}$ then $m$ is ergodic with respect to $\sigma$.

Proof. Let $m=\frac{1}{n} \sum_{i=0}^{n-1} \mu \circ \sigma^{-i}$. Property (1) follows from Abramov's formula and properties (2) and (3) are straightforward calculations.

To find good covers of $X_{\alpha}$ it is helpful to relate $X_{\alpha}$ to sets defined by the behaviour of the ergodic ratio of $\psi$ and $\phi$. If we fix $\alpha \in \mathbb{R}$ then we can use the results in the previous section to obtain the following lemma.

Lemma 5. If $\underline{i} \in \Sigma_{A} \backslash E$, $\Pi \underline{i}=x$ and $x \in X_{\alpha}$ then

$$
\liminf _{n \rightarrow \infty}-\frac{S_{n} \phi(\underline{i})}{S_{n} \psi(\underline{i})}=\alpha
$$

Proof. We combine Lemma 2 and the tempered distortion property to see that there exists a subsequence $\left\{n_{m}\right\}_{m \in \mathbb{N}}$ such that for each $m \in \mathbb{N}$

$$
B\left(x, K^{-1} e^{-2 n_{m} \rho_{n_{m}}-S_{n_{m}} \psi(\underline{i})}\right) \subseteq \Delta_{n}(\underline{i}) .
$$

Thus for $\epsilon>0$ and $m$ sufficiently large we have that

$$
\begin{aligned}
\nu\left(\Delta_{n_{m}}(\underline{i})\right) & \geq \nu\left(B\left(x, K^{-1} e^{-2 n_{m} \rho_{n_{m}}-S_{n_{m}} \psi(\underline{i})}\right)\right) \\
& \geq\left(K^{-1} e^{-2 n_{m} \rho_{n_{m}}-S_{n_{m}} \psi(\underline{i})}\right)^{\alpha+\epsilon}
\end{aligned}
$$

At the same time,

$$
\nu\left(\Delta_{n_{m}}(\underline{i})\right) \leq K e^{n_{m} \rho_{n_{m}}+S_{n_{m}} \phi(\underline{i})} .
$$

Note that $-\frac{1}{n} S_{n} \phi$ is uniformly bounded away from zero. This implies that $\frac{1}{n} S_{n} \psi(\underline{i})$ is bounded away from zero as well, dominating $\rho_{n}$. Hence,

$$
e^{S_{n_{m}} \phi(\underline{i})} \geq K^{-\alpha-\epsilon-1} e^{-(2 \alpha+2 \epsilon+1) n_{m} \rho_{n_{m}}} \cdot e^{-(\alpha+\epsilon) S_{n_{m}} \psi(\underline{i})}
$$

proves the inequality in one direction. The other direction follows from

$$
B\left(x, K e^{n \rho_{n}-S_{n} \psi(\underline{i})}\right) \supseteq \Delta_{n}(\underline{i})
$$

(it holds for all $n$ by the tempered distortion property) in a similar way. 
So if we let

$$
Y_{\alpha}=\left\{\underline{i} \in \Sigma_{A}: \liminf _{n \rightarrow \infty}-\frac{S_{n} \phi(\underline{i})}{S_{n} \psi(\underline{i})}=\alpha\right\}
$$

then we can deduce that

$$
\operatorname{dim}_{H} X_{\alpha} \leq \operatorname{dim}_{H} \Pi Y_{\alpha}
$$

To obtain the upper bound for Theorem 1 we will calculate an upper bound for the dimension of $\Pi Y_{\alpha}$. Let

$Y_{\alpha, \epsilon}=\left\{\underline{i} \in \Sigma_{A}: \alpha-\epsilon \leq-\frac{S_{n} \phi(\underline{i})}{S_{n} \psi(\underline{i})} \leq \alpha+\epsilon\right.$ for infinitely many $\left.n \in \mathbb{N}\right\}$.

Let

$Y_{n, \alpha, \epsilon}=\left\{\left[i_{1}, \ldots, i_{n}\right]: \exists \underline{i} \in\left[i_{1}, \ldots, i_{n}\right]\right.$ with $\left.\left(-\frac{S_{n} \phi(\underline{i})}{S_{n} \psi(\underline{i})}\right) \in(\alpha-\epsilon, \alpha+\epsilon)\right\}$

and note that for all $N \in \mathbb{N}$ we have $\cup_{n \geq N} Y_{n, \alpha, \epsilon} \supseteq Y_{\alpha, \epsilon}$. We now define $s_{n}$ to satisfy

$$
\sum_{\left[i_{1}, \ldots, i_{n}\right] \in Y_{n, \alpha, \epsilon}}\left(D_{n}(\underline{\tilde{i}})\right)^{s_{n}}=1
$$

where $\underline{\tilde{i}}$ denotes some element of the cylinder $\left[i_{1}, \ldots, i_{n}\right] \in Y_{n, \alpha, \epsilon}$. Let

$$
s=\limsup _{n \rightarrow \infty} s_{n}
$$

As $-\frac{1}{n} S_{n} \phi$ is uniformly bounded away from zero, for all cylinders $\left[i_{1}, \ldots, i_{n}\right] \in Y_{n, \alpha, \epsilon}$ we have $-\frac{1}{n} \log D_{n}(\underline{i})$ also bounded away from zero for $\underline{i} \in\left[i_{1}, \ldots, i_{n}\right]$. It is thus clear that for any $\delta>0$ there exists $N>0$ such that

$$
\sum_{n>N} \sum_{Y_{n, \alpha, \epsilon}}\left(D_{n}(\underline{\tilde{i}})\right)^{s+\delta}<1
$$

Consider now for increasing $N$ the unions $\bigcup_{n>N} Y_{n, \alpha, \epsilon}$, each of them forms a covering for $\Pi Y_{\alpha, \epsilon}$. By (3.2), it follows that

$$
\operatorname{dim}_{H} \Pi Y_{\alpha, \epsilon} \leq s .
$$

We now want to relate this value $s$ to the entropy and Lyapunov exponent of an invariant measure. To do this we introduce a class of measures which will not be $\sigma$-invariant but will be $\sigma^{m}$ invariant for some $m$. Let

$$
C_{n}=\left\{\left[i_{1}, \ldots, i_{n}\right]:\left[i_{1}, \ldots, i_{n}\right] \cap \Sigma_{A} \neq \emptyset\right\}
$$


and $q: C_{n} \rightarrow[0,1]$ satisfy $\sum_{\left[i_{1}, \ldots, i_{n}\right] \in C_{n}} q\left(C_{n}\right)=1$. Let $k(n)$ be the smallest integer such that for any $\underline{i}^{n}, \underline{j}^{n} \in C_{n}$ there exists $\omega(\underline{i}, \underline{j}) \in C_{k}$ such that

$$
\left[i_{1}, \ldots, i_{n}, \omega_{1}, \ldots, \omega_{k}, j_{1}, \ldots, j_{n}\right] \cap \Sigma_{A} \neq \emptyset
$$

and

$$
\inf \left\{\exp \left(S_{n+k} \psi(\underline{i}): \underline{i} \in\left[i_{1}, \ldots, i_{n}, \omega_{1}, \ldots, \omega_{k}\right]\right)\right\}>1 .
$$

We can then use our function $q$ to define a $\sigma^{n+k}$ invariant measure $\mu_{q}$. We define the measure on cylinders of level $l n+(l-1) k$ by setting

$$
\mu_{q}\left(\left[\underline{i}_{1}^{n} \omega\left(\underline{i}_{1}^{n}, \underline{i}_{2}^{n}\right) \underline{i}_{2}^{n} \cdots \omega\left(\underline{i}_{l-1}^{n}, \underline{i}_{l}^{n}\right), \underline{i}_{l}^{n}\right]\right)=\prod_{m=1}^{l} q\left(\underline{i}_{m}^{n}\right)
$$

and the measure of all cylinders of level $l n+(l-1) k$ not of this form to be 0 . We will write the space of all such measures as $M_{n}\left(\Sigma_{A}\right)$. The following Lemma describes the behaviour of these measures.

Lemma 6. The family of measures $M_{n}\left(\Sigma_{A}\right)$ satisfies the following:

(1) If $\mu_{q} \in M_{n}\left(\Sigma_{A}\right)$ then $\mu_{q}$ is $\sigma^{n+k}$-invariant and ergodic.

(2) For $\mu_{q} \in M_{n}\left(\Sigma_{A}\right)$ we have that

$$
h\left(\mu_{q}, \sigma^{n+k}\right)=-\sum_{\left[i_{1}, \ldots, i_{n}\right] \in C_{n}} q\left(i_{1}, \ldots, i_{n}\right) \log q\left(i_{1}, \ldots, i_{n}\right)
$$

with the usual convention $0 \log 0=0$.

(3) For any $\mu_{q} \in M_{n}\left(\Sigma_{A}\right)$ the set $\Pi\left(\operatorname{supp} \mu_{q}\right)$ will not contain any parabolic points. In particular the measure $\nu_{q}=\left(\frac{1}{n+k} \sum_{i=0}^{n+k-1} \mu_{q} \circ \sigma^{-i}\right) \circ$ $\Pi^{-1}$ will be uniformly hyperbolic.

(4) The measures $\mu_{q} \in M_{n}\left(\Sigma_{A}\right)$ vary continuously, with $q$, in the weak* topology.

(5) We have

$$
\left|\int \frac{1}{n+k} S_{n+k} \psi d \mu_{q}-\frac{1}{n} \sum_{\underline{i}^{n} \in C_{n}} q\left(\underline{i}^{n}\right) S_{n} \psi(\underline{i})\right| \leq \frac{k L}{n+k}+\rho_{n}
$$

and

$$
\left|\int \frac{1}{n+k} S_{n+k} \phi d \mu_{q}-\frac{1}{n} \sum_{\underline{i}^{n} \in C_{n}} q\left(\underline{i}^{n}\right) S_{n} \phi(\underline{i})\right| \leq \frac{k L}{n+k}+\rho_{n}
$$

where $\underline{i}$ is an arbitrary point from $\left[\underline{i}^{n}\right]$ and $L=\max (\sup |\phi|, \sup \psi)$. 
Proof. Let $n \in \mathbb{N}$ and $q$ be a function defining a measure, $\mu_{q}$, in $M_{n}\left(\Sigma_{A}\right)$. This measure is going to be defined as a $\sigma^{n}$ invariant Bernoulli measure, that is, we take a Bernoulli measure defined on $\left(\{1, \ldots, p\}^{n}\right)^{\mathbb{N}}$ (defined by describing its values on each cylinder $\left[i_{1}, \ldots, i_{n}\right]$ ) and then transport this measure to $\Sigma$ by the usual identification. We set $\eta_{q}$ by

$$
\eta_{q}\left(\left[i_{1}, \ldots, i_{n}\right]\right)=q\left(\left[i_{1}, \ldots, i_{n}\right]\right) \text { if }\left[i_{1}, \ldots, i_{n}\right] \cap \Sigma_{A} \neq \emptyset
$$

and

$$
\eta_{q}\left(\left[i_{1}, \ldots, i_{n}\right]\right)=0 \text { if }\left[i_{1}, \ldots, i_{n}\right] \cap \Sigma_{A}=\emptyset .
$$

We can build a map, $\gamma: \operatorname{supp} \mu_{q} \rightarrow \Sigma$ by

$$
\gamma\left(\underline{i}_{1}^{n} \omega\left(\underline{i}_{1}^{n}, \underline{i}_{2}^{n}\right) \underline{i}_{2}^{n} \cdots \omega\left(\underline{i}_{l-1}^{n}, \underline{i}_{l}^{n}\right) \underline{i}_{l}^{n} \cdots\right)=\underline{i}_{1}^{n} \underline{i}_{2}^{n} \cdots, \underline{i}_{l}^{n} \cdots
$$

which maps $\mu_{q}$ onto $\eta_{q}$. In fact this is an isomorphism of the dynamical systems $\left(\Sigma, \sigma^{n+k}, \mu_{q}\right)$ and $\left(\Sigma, \sigma^{n}, \eta_{q}\right)$. Parts 1 and 2 follow immediately. Part 3 follows from the definition of $\omega$. Part 4 follows from the definition of $\gamma$ and the weak* continuity of Bernoulli measures with respect to the generating vector.

To see part 5 we note that

$$
\left|\int \frac{1}{n} S_{n} \psi d \mu_{q}-\frac{1}{n} \sum_{\underline{i}^{n} \in C_{n}} q\left(\underline{i}^{n}\right) S_{n} \psi(\underline{i})\right| \leq \rho_{n}
$$

and

$$
\left|\int \frac{1}{n} S_{n} \phi d \mu_{q}-\frac{1}{n} \sum_{\underline{i}^{n} \in C_{n}} q\left(\underline{i}^{n}\right) S_{n} \phi(\underline{i})\right| \leq \rho_{n}
$$

follow from tempered distortion. The assertion now follows by direct calculation.

The importance of this family of measures is that certain measures in this family can be related to $s$, the upper bound for $\operatorname{dim}_{H} X_{\alpha}$.

Lemma 7. We can find a sequence of measures $\mu_{n} \in M_{n}\left(\Sigma_{A}\right)$ such that

$$
\alpha-\epsilon \leq \liminf _{n \rightarrow \infty}-\frac{\int S_{n} \phi d \mu_{n}}{\int S_{n} \psi d \mu_{n}} \leq \limsup _{n \rightarrow \infty}-\frac{\int S_{n} \phi d \mu_{n}}{\int S_{n} \psi d \mu_{n}} \leq \alpha+\epsilon,
$$

and

$$
\lim _{n \rightarrow \infty}\left(\frac{h\left(\mu_{n}, \sigma^{n+k}\right)}{\int S_{n} \psi d \mu_{n}}-s_{n}\right)=0 .
$$

Those measures are generated with $q$ supported in $Y_{n, \alpha, \epsilon}$. 
Proof. Given $\underline{i}^{n} \in C_{n}$, we let $q\left(\underline{i}^{n}\right)=\left(D_{n}(\underline{i})\right)^{s_{n}}$ if $\underline{i}^{n} \in Y_{n, \alpha, \epsilon}$ and $q\left(\underline{i}^{n}\right)=$ 0 otherwise. We consider the corresponding measure $\mu_{q} \in M_{n}$. Let us estimate the relevant parameters for the measure $\mu_{q}$. As $\frac{1}{n} S_{n} \phi$ is bounded away from zero, the definition of $Y_{n, \alpha, \epsilon}$ implies that $\frac{1}{n} S_{n} \psi$ is bounded away from zero as well, hence by Lemma 6, statement 5

hence

$$
\alpha-\epsilon-o(1) \leq-\frac{\int S_{n+k} \phi d \mu_{q}}{\int S_{n+k} \psi d \mu_{q}} \leq \alpha+\epsilon+o(1)
$$

$$
\alpha-\epsilon-o(1)-O(1 / n) \leq-\frac{\int S_{n} \phi d \mu_{q}}{\int S_{n} \psi d \mu_{q}} \leq \alpha+\epsilon+o(1)+O(1 / n),
$$

which proves the first part of the assertion. We also have that

$$
h\left(\mu_{q}, \sigma^{n+k}\right)=-s_{n} \sum_{\underline{i}^{n} \in Y_{n, \alpha, \epsilon}}\left(D_{n}\left(\underline{i}^{n}\right)\right)^{s_{n}} \log D_{n}\left(\underline{i}^{n}\right) .
$$

On the other hand, by combining Lemma 1 and Lemma [6] statement 5 we can deduce that

$$
\left|\int \frac{1}{n} S_{n} \psi d \mu_{q}+\frac{1}{n} \sum_{\underline{i}^{n} \in Y_{n, \alpha, \epsilon}}\left(D_{n}\left(\underline{i}^{n}\right)\right)^{s_{n}} \log D_{n}\left(\underline{i}^{n}\right)\right| \leq\left|\int \psi d \mu_{q}-\frac{1}{n} \sum_{\underline{i}^{n} \in Y_{n, \alpha, \epsilon}} q\left(\underline{i}^{n}\right) S_{n} \psi(\underline{i})\right|+\rho_{n} \rightarrow 0 .
$$

As the left hand side of (3.4) is a difference of two terms, the first of which is bounded away from zero by (3.3), the second term is bounded as well. The second part of assertion follows. lary.

By combining this lemma with Lemma 4 we get an immediate corol-

Corollary 1. We can find a sequence of ergodic measures $\mu_{n} \in \mathcal{M}_{\sigma}\left(\Sigma_{A}\right)$ such that

$$
\alpha-\epsilon \leq \lim _{n \rightarrow \infty}-\frac{\int \phi d \mu_{n}}{\lambda\left(\mu_{n}\right)} \leq \alpha+\epsilon
$$

and

$$
\limsup _{n \rightarrow \infty} \frac{h\left(\mu_{n}, \sigma\right)}{\int \psi d \mu_{n}} \geq s .
$$

Moreover these measures are uniformly hyperbolic.

Proof. We consider $\mu_{q} \in M_{n}\left(\Sigma_{A}\right)$ and choose a subsequence for which the limit of $\int \phi d \mu_{q_{i}} / \int \psi d \mu_{q_{i}}$ exists. By Lemma 4 we can find an ergodic measure $\tilde{\mu} \in \mathcal{M}_{\sigma}\left(\Sigma_{A}\right)$ such that $h(\tilde{\mu})=\frac{1}{n+k} h\left(\mu_{q}, \sigma^{n+k}\right), \int \psi \mathrm{d} \tilde{\mu}=$ 
$\frac{1}{n+k} \int S_{n+k} \psi \mathrm{d} \mu_{q}$ and $\int \phi \mathrm{d} \tilde{\mu}=\frac{1}{n+k} \int S_{n+k} \phi \mathrm{d} \mu_{q}$. Since $k$ is independent of $n$ the result easily follows from Lemma 7.

In the proof of Theorem 2 we will need the sequence $s_{m}$ to be convergent. This can be deduced from Lemma 7 .

Corollary 2. In the above construction we have that $s=\lim _{\sup } \sin _{n \rightarrow \infty} s_{n}=$ $\lim _{n \rightarrow \infty} s_{n}$. In other words the sequence $s_{n}$ is convergent.

Proof. Consider the measure $\nu_{n}=\mu_{n} \circ \Pi^{-1}$, where $\mu_{n}$ is defined in Corollary 1. As it is ergodic, its Hausdorff dimension equals its local dimension at a typical point. By Lemma 3 at a typical point its local dimension is equal to its symbolic local dimension $\lim \log \nu_{n}\left(\Delta_{n}(\underline{i})\right) / \log D_{n}(\underline{i})$. By Birkhoff Ergodic Theorem, there exists some $N$ such that for all $m>N$ the $m$-th level cylinders $\left[i_{1}, \ldots, i_{m}\right]$ for which

$$
D_{m}(\underline{i})^{\operatorname{dim}_{H} \nu_{n}-\epsilon}>\mu_{n}\left(\left[i_{1}, \ldots, i_{m}\right]\right)
$$

have total measure $\mu_{n}$ greater than $2 / 3$.

As $\mu_{n}$ is ergodic, for $\mu_{n}$-almost every $\underline{i}\left[i_{1}, \ldots, i_{m}\right] \in Y_{m, \alpha, 2 \epsilon}$ for $m$ big enough. This implies that there exists $N$ such that for all $m>N$ the cylinders from $Y_{m, \alpha, 2 \epsilon}$ have total measure $\mu_{n}$ greater than $2 / 3$.

Combining those two statements, we see that for all $m$ big enough

$$
\sum_{\left[i_{1}, \ldots, i_{m}\right] \in Y_{m, \alpha, 2 \epsilon}}\left(D_{m}(\underline{i})\right)^{\operatorname{dim}_{H} \nu_{n}-\epsilon}>1 / 3 .
$$

Since the diameters of the cylinders from $Y_{m, \alpha, 2 \epsilon}$ are exponentially small (3.5) implies that

$$
s_{m} \geq \operatorname{dim}_{H} \nu_{n}-\epsilon-O(1 / m)
$$

for all sufficiently big $m$. At the same time, by [8]

$$
\operatorname{dim}_{H} \nu_{n}=\frac{h\left(\mu_{n}, \sigma\right)}{\int \psi d \mu_{n}}
$$

which, by Corollary 1, can be chosen arbitrarily close to $s$.

\section{Proof of Theorem 1}

The first statement follows immediately from Corollary 1. The second and fourth statements are going to be obtained in the course of the proof of the main, third statement. We will first prove the easy upper bound. 
It follows immediately from Corollary 1 that for any $\epsilon>0$

$\operatorname{dim}_{H} \Pi Y_{\alpha} \leq \sup _{\mathcal{M}_{H}\left(\Sigma_{A}\right)}\left\{\frac{h(\mu, \sigma)}{\lambda(\mu)}: \alpha-\epsilon \leq-\frac{\int \phi \mathrm{d} \mu}{\lambda(\mu)} \leq \alpha+\epsilon\right.$ and $\left.\lambda(\mu)>0\right\}$.

Note that since $\mathcal{M}_{H}\left(\Sigma_{A}\right) \subset \mathcal{M}_{\sigma\left(\Sigma_{A}\right)}$ we also have the same inequality if the supremum is taken over all invariant measures,

$\operatorname{dim}_{H} \Pi Y_{\alpha} \leq \sup _{\mathcal{M}_{\sigma}\left(\Sigma_{A}\right)}\left\{\frac{h(\mu, \sigma)}{\lambda(\mu)}: \alpha-\epsilon \leq-\frac{\int \phi \mathrm{d} \mu}{\lambda(\mu)} \leq \alpha+\epsilon\right.$ and $\left.\lambda(\mu)>0\right\}$.

To complete the proof of the upper bound we need to show the supremum over invariant measures

$$
\sup _{\mu \in \mathcal{M}_{\sigma}\left(\Sigma_{A}\right)}\left\{\frac{h(\mu, \sigma)}{\lambda(\mu)}:-\frac{\int \phi \mathrm{d} \mu}{\lambda(\mu)}=\alpha \text { and } \lambda(\mu)>0\right\}
$$

varies continuously with $\alpha$.

The supremum is an upper semi-continuous function of $\alpha$ because of the upper semi-continuity of entropy (see Theorem 8.2 in [22]) and the continuity of the Lyapunov exponent. We now fix $\alpha<\beta \in$ $\left[\alpha_{\min }, \alpha_{\max }\right] \backslash\{\infty\}$ and let $\mu_{\alpha}, \mu_{\beta} \in \mathcal{M}_{\sigma}\left(\Sigma_{A}\right)$ satisfy that $-\frac{\int \phi \mathrm{d} \mu_{\alpha}}{\lambda\left(\mu_{\alpha}\right)}=\alpha$, $-\frac{\int \phi \mathrm{d} \mu_{\beta}}{\lambda\left(\mu_{\beta}\right)}=\beta, \frac{h\left(\mu_{\alpha}, \sigma\right)}{\lambda\left(\mu_{\alpha}\right)}=\operatorname{dim}_{H} X_{\alpha}$ and $\frac{h\left(\mu_{\beta}, \sigma\right)}{\lambda\left(\mu_{\beta}\right)}=\operatorname{dim}_{H} X_{\beta}$ (those measures exist because $\alpha<\beta<\infty$ implies $\left.\lambda\left(\mu_{\alpha}\right), \lambda\left(\mu_{\beta}\right) \geq c(\beta)>0\right)$. To show that the function is right lower semi-continuous at $\alpha$ and left lower semi-continuous at $\beta$ we simply consider convex combinations of $\mu_{\alpha}$ and $\mu_{\beta}$.

We now turn to the lower bound. Initially we will prove the lower bound in the case where there exist hyperbolic measures with dimension arbitrarily close to that of $\Lambda$. To complete the proof we will then need to show that $\operatorname{dim}_{H} X_{\infty}=\operatorname{dim}_{H} \Lambda$ still holds in the case when such hyperbolic measures are not known to exist. Note that if $T$ is $C^{1+\theta}$ then such measures always exist and that it is unknown whether this is the case if $T$ is merely $C^{1}$.

We begin with the following lemma:

Lemma 8. Let $\underline{i} \in \Sigma_{A} \backslash E, x=\Pi \underline{i}$ and $\alpha \in \mathbb{R} \cup\{\infty\}$. If

$$
\lim _{n \rightarrow \infty}-\frac{S_{n} \phi(\underline{i})}{S_{n} \psi(\underline{i})}=\alpha
$$

and

$$
\lim _{n \rightarrow \infty} \frac{1}{n} \log \left(\frac{Z_{n}(\underline{i})}{D_{n}(\underline{i})}\right)=0
$$


then

$$
\lim _{r \rightarrow 0} \frac{\log \nu(B(x, r))}{\log r}=\alpha .
$$

Proof. Let $\underline{i} \in \Sigma_{A} \backslash E$ satisfy the hypothesis in the Lemma with $\alpha$ finite and let $x=\Pi \underline{i}$. Fix $\epsilon, r>0$ and choose $n$ such that

$$
D_{n}(\underline{i})<r \leq D_{n-1}(\underline{i}) .
$$

It follows that

$$
B(x, r) \supset \Delta_{n}(\underline{i})
$$

and if $r$ is chosen to be sufficiently small then

$$
\nu(B(x, r)) \geq\left(D_{n-1}(\underline{i})\right)^{\alpha+\epsilon} .
$$

Furthermore, by property (4.1) for $r$ small enough

$$
B\left(x, r^{1+\epsilon}\right) \subset \Delta_{n}(\underline{i})
$$

and hence

$$
\nu\left(B\left(x, r^{1+\epsilon}\right)\right) \leq\left(D_{n}(\underline{i})\right)^{\alpha-\epsilon} \leq r^{\alpha-\epsilon} .
$$

Since $\epsilon$ can be chosen to be arbitrarily small, it follows that

$$
\lim _{r \rightarrow 0} \frac{\log \nu(B(x, r))}{\log r}=\alpha .
$$

For $\alpha=\infty$ we fix $\epsilon, r>0$ and choose $n$ in the same way. For any $\beta>0$ if $r$ is sufficiently small then we have

$$
\begin{aligned}
\nu\left(B\left(x, r^{1+\epsilon}\right)\right) & \leq \tilde{\nu}\left(\left[i_{1}, \ldots, i_{n}\right]\right) \\
& \leq\left(D_{n}(\underline{i})\right)^{\beta} \leq r^{\beta}
\end{aligned}
$$

and the result follows.

By Lemma 3, the assumptions of Lemma 8 are satisfied for almost all points for any ergodic measure $\mu$ on $\Sigma_{A}$, with

$$
\alpha=-\frac{\int \phi d \mu}{\lambda(\mu)} .
$$

Thus, almost all points for measure $\mu \circ \Pi^{-1}$ will belong to $X_{\alpha}$, and by [8] the Hausdorff dimension of this set is $h(\mu, \sigma) / \lambda(\mu)$. Hence,

$$
\operatorname{dim}_{H} X_{\alpha} \geq \sup \left\{\frac{h(\mu, \sigma)}{\lambda(\mu)}: \mu \text { ergodic, }-\frac{\int \phi d \mu}{\lambda(\mu)}=\alpha\right\} .
$$

However, what we need to prove is the lower bound given by supremum over all invariant measures, not only ergodic measures in (4.2). The first case is $\alpha \in\left\{\alpha_{\min }, \alpha_{\max }\right\}$, and we will prove that for such $\alpha$ suprema over ergodic measures and over invariant measures are equal. 
For $\alpha=\alpha_{\min }$ we consider any invariant measure $\mu$ such that

$$
-\frac{\int \phi \mathrm{d} \mu}{\int \psi \mathrm{d} \mu}=\alpha_{\min } .
$$

For any measure $\mu_{i}$ in the ergodic decomposition of $\mu$ it follows that

$$
-\frac{\int \phi \mathrm{d} \mu_{i}}{\int \psi \mathrm{d} \mu_{i}}=\alpha_{\min }
$$

since otherwise there would exist an ergodic measure contradicting the definition of $\alpha_{\min }$. Moreover at least one measure in the ergodic decomposition must satisfy $\frac{h\left(\mu_{i}, \sigma\right)}{\lambda\left(\mu_{i}\right)} \geq \frac{h(\mu, \sigma)}{\lambda(\mu)}$. This completes the proof of the lower bound for $\alpha=\alpha_{\min }$.

As the same proof works for $\alpha=\alpha_{\max }<\infty$, there are two cases left: $\alpha \in\left(\alpha_{\min }, \alpha_{\max }\right)$ and $\alpha=\alpha_{\max }=\infty$. This time we will not be able to find a good measure for the given $\alpha$, but we will find them for some close $\alpha$. In fact, the measures we are going to find will be Gibbs measures, hence in particular ergodic. We will then use them to construct certain big set and then prove that it is contained in $X_{\alpha}$. The beginning parts of the proofs differ in both cases but the subsequent argument is the same.

Let $\mu$ be an invariant measure satisfying $-\int \phi d \mu / \lambda(\mu)=\alpha \in\left(\alpha_{\min }, \infty\right)$. By Lemma 4.2 from [15], there exists a sequence of Gibbs measures $\mu_{i}$ weakly converging to $\mu$, such that $h\left(\mu_{i}, \sigma\right) \rightarrow h(\mu, \sigma)$. Note these are not just weak Gibbs measures but Gibbs measures in the usual sense and thus ergodic. The weak convergence implies the convergence of $\int \phi$ and of $\lambda$ which means that $h\left(\mu_{i}, \sigma\right) / \lambda\left(\mu_{i}\right) \rightarrow h(\mu, \sigma) / \lambda(\mu)$ and so the Hausdorff dimension of the measures $\mu_{i} \circ \Pi^{-1}$ converges to this limit.

In the case where $\alpha=\infty$ we will let

$$
\eta=\sup _{\mu \in \mathcal{M}_{\sigma}\left(\Sigma_{A}\right)}\left\{\frac{h(\mu, \sigma)}{\lambda(\mu)}: \lambda(\mu)>0\right\} .
$$

Our initial goal here will be to prove that $\operatorname{dim}_{H} X_{\infty} \geq \eta$. By lemma 4.2 from [15] it follows that for any $\epsilon>0$ we can find an ergodic measure $\mu_{1}$ such that $\operatorname{dim}_{H} \mu_{1} \geq \eta-\epsilon$. If we take a parabolic invariant measure $\mu_{2}$ and consider invariant measures of the form $q \mu_{1}+(1-q) \mu_{2}$ for $0<q<1$ then it is clear that

$$
\frac{h\left(q \mu_{1}+(1-q) \mu_{2}, \sigma\right)}{\lambda\left(q \mu_{1}+(1-q) \mu_{2}\right)}=\frac{h\left(\mu_{1}, \sigma\right)}{\lambda\left(\mu_{1}\right)}
$$

and that $\lim _{q \rightarrow 0} \lambda\left(q \mu_{1}+(1-q) \mu_{2}\right)=0$. Thus again applying Lemma 4.2 from [15] to these measures we get a sequence of Gibbs measures 
$\mu_{j}$ such that

$$
\lim _{j \rightarrow \infty} \frac{h\left(\mu_{j}, \sigma\right)}{\lambda\left(\mu_{j}\right)} \geq \eta-\epsilon
$$

and

$$
\lim _{j \rightarrow \infty}-\frac{\int \phi d \mu_{j}}{\lambda\left(\mu_{j}\right)}=\infty .
$$

Hence, in both cases we have a sequence of Gibbs measures $\mu_{i}$ for which $-\int \phi d \mu_{i} / \lambda\left(\mu_{i}\right) \rightarrow \alpha$ and we want to prove that

$$
\operatorname{dim}_{H} X_{\alpha} \geq \liminf \frac{h\left(\mu_{i}, \sigma\right)}{\lambda\left(\mu_{i}\right)} .
$$

The aim will be to construct a (non-invariant) measure $\tilde{\mu}$ such that $\lim _{n \rightarrow \infty} \frac{1}{n} S_{n} \phi(\underline{i})=\lim _{n \rightarrow \infty} \int \phi d \mu_{n}, \lim _{n \rightarrow \infty} \frac{1}{n} S_{n} \psi(\underline{i})=\lim _{n \rightarrow \infty} \lambda\left(\mu_{n}\right)$ for $\tilde{\mu}$-almost all $\underline{i}, \operatorname{dim}_{H} \tilde{\mu} \geq \lim _{n \rightarrow \infty} \operatorname{dim}_{H} \mu_{n}$ and the statement of Lemma 3 is satisfied. Here we assume (by restricting to a subsequence of $\left\{\mu_{n}\right\}$ if necessary) that the limits of $\int \phi d \mu_{n}, \int \psi d \mu_{n}$ exist. The measure $\tilde{\mu}$ will be constructed using the approach from section 5 of [6].

Let $\left\{m_{i}\right\}$ be an increasing sequence of integers, $m_{0}=0$. We will define a new measure $\tilde{\mu}$ (w-measure in the terminology of [6]) inductively: $\tilde{\mu}(\Lambda)=1$ and

$$
\tilde{\mu}\left(\Delta_{\underline{i}^{m_{i}} \underline{j}^{m_{i+1}-m_{i}}}\right)=c_{i+1}\left(\underline{i}^{m_{i}}\right) \tilde{\mu}\left(\Delta_{\underline{i}^{m_{i}}}\right) \Pi_{*} \mu_{i+1}\left(\Delta_{\underline{j}^{m_{i+1}-m_{i}}}\right)
$$

where $c_{i+1}\left(\underline{i}^{m_{i}}\right)$ are normalizing constants. In other words, this measure is concentrated on points whose trajectory for some time $m_{1}$ is distributed according to measure $\mu_{1}$, then for time $\left(m_{2}-m_{1}\right)$ it is distributed according to $\mu_{2}$ and so on.

The properties of measures of this type were checked in [6]. First, as proved in [6], Proposition 9, the Hausdorff dimension of $\mu$ is not smaller than the lower limit of Hausdorff dimensions of $\mu_{i}$ provided that $m_{i}$ grow quickly enough. Furthermore, there exists a sequence $\left\{K_{i}\right\}$ with each $K_{i}$ depending only on $\mu_{i}$ such that for each $m \in\left(m_{i}, m_{i+1}\right)$

$$
K_{i+1}^{-1} \leq \frac{\tilde{\mu}\left(\Delta_{\underline{i}^{m_{i}} \underline{j}^{m-m_{i}}}\right)}{\tilde{\mu}\left(\Delta_{\underline{i}^{m_{i}}}\right) \mu_{i+1}\left(\Delta_{\underline{j}^{m-m_{i}}}\right)} \leq K_{i+1} .
$$

An immediate consequence of (4.3) is that for any bounded potential (in particular, $\phi$ or $\psi$ ), if $m_{i}$ grows quickly enough then its Cesaro average at a $\tilde{\mu}$ typical point is equal to $\lim \int \phi d \mu_{i}$ (see Proposition 9 in [6] again). In our setting this means that,

$$
\frac{S_{n} \phi(\underline{i})}{S_{n} \psi(\underline{i})} \rightarrow \alpha
$$

$\tilde{\mu}$-almost everywhere. 
To finish we need to use this to prove that $\tilde{\mu}\left(X_{\alpha}\right)=1$ which we will do by showing the statement of Lemma 3 is valid for $\tilde{\mu}$ which will allow us to apply Lemma 8. Indeed, all the $\mu_{i}$ satisfy Lemma 3. Hence, for every $\epsilon$ there exists a sequence $\left\{C_{i}\right\}_{i \in \mathbb{N}}$ such that

$$
\mu_{i}\left(\left\{\underline{j} \in \Sigma_{A}: \exists k \text { where } Z_{k}(\underline{j})<C_{i} D_{k}(\underline{i}) e^{-k \epsilon}\right\}\right)<K_{i+1}^{-1} \cdot 2^{-i} .
$$

Due to (4.3), this implies that for every $i$ and $\underline{i}^{m_{i}}$, inside every cylinder $\left[i_{1}, \ldots, i_{m_{i}}\right]$ the relative $\tilde{\mu}$ measure of points $\underline{j}$ for which there exists $k \in\left(m_{i}, m_{i+1}\right]$ such that

$$
Z_{k}(\underline{j})<C_{i+1} D_{k}(\underline{j}) e^{-k \epsilon}
$$

is not greater than $2^{-i-1}$. By the Borel-Cantelli Lemma, (4.5) is satisfied only finitely many times for $\tilde{\mu}$-almost any $\underline{j}$.

Note here that $D_{k}(j)$ is decreasing exponentially fast with $k$ for $\mu_{i^{-}}$ almost every $\underline{j}$ (but the rate depends on $i$ ). Thus, if we choose $\left\{m_{i}\right\}$ to be increasing quickly enough, we will get

$$
\liminf _{k \rightarrow \infty} \frac{1}{k} \log \left(\frac{Z_{k}(\underline{j})}{D_{k}(\underline{j})}\right) \geq-2 \epsilon
$$

$\tilde{\mu}$-almost everywhere. As this holds for any $\epsilon>0$ we know that

$$
\lim _{k \rightarrow \infty} \frac{1}{k} \log \left(\frac{Z_{k}(\underline{j})}{D_{k}(\underline{j})}\right)=0
$$

and so we can use Lemma 8 to deduce that $\tilde{\mu}\left(X_{\alpha}\right)=1$. We have shown that for $\alpha \in\left(\alpha_{\min }, \alpha_{\max }\right)$ we have that

$$
\operatorname{dim}_{H} X_{\alpha} \geq \sup _{\mu \in \mathcal{M}_{\sigma}\left(\Sigma_{A}\right)}\left\{\frac{h(\mu, \sigma)}{\lambda(\mu)}:-\frac{\int \phi \mathrm{d} \mu}{\lambda(\mu)}=\alpha \text { and } \lambda(\mu)>0\right\}
$$

and if $\alpha_{\max }=\infty$ then

$$
\operatorname{dim}_{H} X_{\infty} \geq \eta
$$

To complete the proof of the lower bound we now need to address the possible case, where $\eta<\operatorname{dim}_{H} \Lambda$.

We start by looking at the local Lyapunov exponents of points. Let

$$
Z=\left\{\underline{i}: \limsup _{n \rightarrow \infty} \frac{S_{n} \psi(\underline{i})}{n}=0\right\}
$$

Lemma 9. We have that

$$
\operatorname{dim}_{H}(\Lambda \backslash \Pi Z) \leq \eta
$$

and thus if $\eta<\operatorname{dim}_{H} \Lambda$ then

$$
\operatorname{dim}_{H} \Pi Z=\operatorname{dim}_{H} \Lambda .
$$


Proof. The first part of this Lemma can easily be deduced from the previous section: the argument estimating the dimension of $\Pi Y_{\alpha}$ also shows that

$\operatorname{dim}_{H} \bigcup_{\alpha \in\left(q_{1}, q_{2}\right)} \Pi Y_{\alpha} \leq \sup _{\mu \in \mathcal{M}_{\sigma}\left(\Sigma_{A}\right)}\left\{\frac{h(\mu, \sigma)}{\lambda(\mu)}: q_{1}-\epsilon \leq-\frac{\int \phi \mathrm{d} \mu}{\lambda(\mu)} \leq q_{2}+\epsilon\right.$ and $\left.\lambda(\mu)>0\right\}$.

The second part then follows immediately.

We now look at points which may have zero local Lyapunov exponent but not have infinite local dimension. Let

$$
L_{\beta}=\Pi\left\{\underline{i} \in Z: Z_{n}(\underline{i}) \leq e^{-n \beta} \text { infinitely often }\right\} .
$$

It is clear that if $\underline{i} \in Z$ but $\Pi(\underline{i}) \notin L_{\beta}$ for any $\beta$ then $\Pi(\underline{i}) \in X_{\infty}$. Thus to complete the proof it suffices to show that $\operatorname{dim}_{H} L_{\beta}$ is bounded away from $\operatorname{dim}_{H} \Lambda$ for all $\beta>0$.

Lemma 10. For any $\beta>0$ we have that $\operatorname{dim}_{H} L_{\beta}=0$.

Proof. Fix $\delta>0$ and let $N_{0}$ be large enough such that $\rho_{n}<\delta$ for $n \geq N_{0}$. Given $n \geq N_{0}$ let

$$
C_{n}=\left\{\left[i_{1}, \ldots, i_{n}\right]: \exists \underline{i} \in\left[i_{1}, \ldots, i_{n}\right] \text { where } S_{n} \psi(\underline{i}) \leq \delta n\right\} .
$$

It follows that the diameter of the projection of each cylinder in $C_{n}$ is at least $e^{-2 \delta n}$ and so there can be at most $e^{2 \delta n}$ cylinders in the set $C_{n}$. Furthermore we have that

$$
Z \subset \bigcap_{N>N_{0}} \bigcup_{n \geq N} \bigcup_{I \in C_{n}} \Pi I
$$

Given $I \in C_{n}$, span $\Pi I=[x, y]$ let $I^{-}=\left[x, x+e^{-\beta n}\right]$ and $I^{+}=$ $\left[y-e^{-\beta n}, y\right]$. We have

$$
L_{\beta} \subset \bigcap_{N>N_{0}} \bigcup_{n \geq N} \bigcup_{I \in C_{n}}\left(I^{-} \cup I^{+}\right)
$$

The union $\bigcup_{I \in C_{n}}\left(I^{-} \cup I^{+}\right)$has at most $2 e^{2 \delta n}$ intervals of length $e^{-\beta n}$ each. Hence,

$$
H_{e^{-\beta n}}^{\frac{2 \delta}{\beta}+\epsilon}\left(L_{\beta}\right) \leq 2 \sum_{n \geq N} \sum_{I \in C_{n}} e^{-n\left(\frac{2 \delta \beta}{\beta}\right)} e^{-n \beta \epsilon} \leq 2 \sum_{n \geq N} e^{-n \beta \epsilon},
$$

where $H_{\epsilon}^{s}$ denotes the approximation of $s$-dimensional Hausdorff measure using $\epsilon$-covers, and thus $\operatorname{dim}_{H} L_{\beta} \leq \frac{2 \delta}{\beta}$. Since $\delta$ was chosen arbitrarily the proof is complete. 
It immediately follows that $\operatorname{dim}_{H} X_{\infty}=\operatorname{dim}_{H} \Lambda$.

To complete the proof of part 4 of the theorem we need to show that $\operatorname{dim}_{H} X_{\alpha}$ is nondecreasing in the presence of parabolic points. Let $\nu_{0}$ be a Dirac measure on a parabolic orbit. Then for any invariant measure $\mu$ measure $\nu_{t}=t \mu+(1-t) \nu_{0}$ is also invariant and satisfies

$$
\frac{h\left(n u_{t}, \sigma\right)}{\lambda\left(\nu_{t}\right)}=\frac{h(\mu, \sigma)}{\lambda(\mu)} \text {. }
$$

Furthermore

$$
-\frac{\int \phi d \nu_{t}}{\int \psi d \nu_{t}}=-\frac{\int \phi d \mu}{\int \psi d \mu}-\frac{1-t}{t} \frac{\int \phi d \nu_{0}}{\int \psi d \mu}
$$

and the right hand side can take any value between $-\frac{\int \phi d \mu}{\int \psi d \mu}$ and $\infty$. The statement follows.

\section{Proof of Theorem 2}

We only need to prove that

$$
\operatorname{dim}_{H} X_{\alpha} \leq \sup _{\mu \in \mathcal{M}_{H}\left(\Sigma_{A}\right)}\left\{\frac{h(\mu, \sigma)}{\lambda(\mu)}:-\frac{\int \phi \mathrm{d} \mu}{\lambda(\mu)}=\alpha\right\}
$$

since the opposite inequality follows from (4.2). For any $\alpha \in\left(\alpha_{\min }, \infty\right)$ we choose $\epsilon<\left(\alpha-\alpha_{\text {min }}\right) / 3$. By Lemma 7, Theorem 1 and Corollary 2 we know that for $n$ sufficiently large there exists measures $\mu_{q_{0}, n}, \mu_{q_{1}, n} \in$ $M_{n}\left(\Sigma_{A}\right)$ with $q_{0}, q_{1}$ of disjoint supports such that

$$
\begin{gathered}
-\frac{\int S_{n+k} \phi \mathrm{d} \mu_{q_{1}, n}}{\int S_{n+k} \psi \mathrm{d} \mu_{q_{1}, n}} \in(\alpha, \alpha+2 \epsilon) \\
\frac{h\left(\mu_{q_{1}, n}, \sigma^{n+k}\right)}{\int S_{n+k} \psi \mathrm{d} \mu_{q_{1}, n}} \geq \operatorname{dim}_{H} X_{\alpha+\epsilon}-\epsilon
\end{gathered}
$$

and

$$
\begin{gathered}
-\frac{\int S_{n+k} \phi \mathrm{d} \mu_{q_{0}, n}}{\int S_{n+k} \psi \mathrm{d} \mu_{q_{0}, n}} \in(\alpha-2 \epsilon, \alpha) \\
\frac{h\left(\mu_{q_{0}, n}, \sigma^{n+k}\right)}{\int S_{n+k} \psi \mathrm{d} \mu_{q_{0}, n}} \geq \operatorname{dim}_{H} X_{\alpha-\epsilon}-\epsilon .
\end{gathered}
$$

For $0 \leq t \leq 1$ we can define $q_{t}=t q_{1}+(1-t) q_{0}$ and consider the measures $\mu_{q_{t}, n} \in M_{n}\left(\Sigma_{A}\right)$. From the properties of $M_{n}\left(\Sigma_{A}\right)$ (Lemma 6 statement 4) it is clear that

$$
-\frac{\int S_{n+k} \phi \mathrm{d} \mu_{q_{t}, n}}{\int S_{n+k} \psi \mathrm{d} \mu_{q_{t}, n}}
$$


will vary continuously with $t$. Thus there will be a $t_{0}$ such that

$$
-\frac{\int S_{n+k} \phi \mathrm{d} \mu_{q_{t_{0}, n}}}{\int S_{n+k} \psi \mathrm{d} \mu_{q_{t_{0}, n}}}=\alpha .
$$

As $q_{0}$ and $q_{1}$ have disjoint supports it follows from the second part of Lemma 6 that

$$
\begin{aligned}
h\left(\mu_{q_{t}, n}, \sigma^{n+k}\right) & =\operatorname{th}\left(\mu_{q_{1}, n}, \sigma^{n+k}\right)+(1-t) h\left(\mu_{q_{0}, n}, \sigma^{n+k}\right)-t \log t-(1-t) \log (1-t) \\
& \geq \operatorname{th}\left(\mu_{q_{1}, n}, \sigma^{n+k}\right)+(1-t) h\left(\mu_{q_{0}, n}, \sigma^{n+k}\right) .
\end{aligned}
$$

At the same time,the fifth part of Lemma 6 implies that

$$
\begin{aligned}
& \left|\int \frac{1}{n+k} S_{n+k} \psi d \mu_{q_{t}, n}-t \int \frac{1}{n+k} S_{n+k} \psi d \mu_{q_{1}, n}-(1-t) \int \frac{1}{n+k} S_{n+k} \psi d \mu_{q_{0}, n}\right| \\
& <\frac{2 k L}{n+k}+2 \rho_{n} .
\end{aligned}
$$

Hence

$$
\limsup _{n \rightarrow \infty}\left(\inf _{t \in(0,1)} \frac{h\left(\mu_{q_{t}, n}, \sigma^{n+k}\right)}{\int S_{n+k} \psi \mathrm{d} \mu_{q_{t}, n}}-\min \left(\frac{h\left(\mu_{q_{1}}, \sigma^{n+k}\right)}{\int S_{n+k} \psi \mathrm{d} \mu_{q_{1}, n}}, \frac{h\left(\mu_{q_{0}, n}, \sigma^{n+k}\right)}{\int S_{n+k} \psi \mathrm{d} \mu_{q_{0}, n}}\right)\right) \geq 0 .
$$

The result now follows by using the continuity of $\operatorname{dim}_{H} X_{\alpha}$ and applying Lemma 4, like in Corollary 1,

\section{Proof of Theorem 3}

Points 1) and 2) of Theorem 3 are an immediate consequence of the following lemmas.

Lemma 11. Let $\mu_{\alpha}$ be an equilibrium state for the potential $\psi_{a}$ and let

$$
\alpha=-\frac{\int \phi d \mu_{\alpha}}{\lambda\left(\mu_{\alpha}\right)} .
$$

We then have that

$$
\frac{h\left(\mu_{\alpha}\right)}{\lambda\left(\mu_{\alpha}\right)}=\sup _{\mu \in \mathcal{M}_{\sigma}\left(\Sigma_{A}\right)}\left\{\frac{h(\mu, \sigma)}{\lambda(\mu)}:-\frac{\int \phi d \mu}{\lambda(\mu)}=\alpha\right\}=b(a) \alpha-a .
$$

Proof. We are going to compare $h / \lambda$ for $\mu_{\alpha}$ and for any other invariant measure $\mu$ with $-\int \phi d \mu / \lambda(\mu)=\alpha$. As $\alpha$ is finite, $\lambda(\mu)$ must be positive. By the variational principle we have that

$$
0=P\left(\psi_{a}\right)=h\left(\mu_{\alpha}, \sigma\right)+\int \psi_{a} d \mu_{\alpha} \geq h(\mu, \sigma)+\int \psi_{a} d \mu .
$$

Dividing by $\lambda\left(\mu_{\alpha}\right)$ or by $\lambda(\mu)$, we get

$$
0=\frac{h\left(\mu_{\alpha}, \sigma\right)}{\lambda\left(\mu_{\alpha}\right)}+a-b(a) \alpha
$$


and

$$
0 \geq \frac{h(\mu, \sigma)}{\lambda(\mu)}+a-b(a) \alpha
$$

from which the assertion follows.

Lemma 12. For any $\alpha \in\left(\alpha_{\min }, \infty\right)$ there exists some measure $\mu_{\alpha}$, which is an equilibrium state for $\psi_{a}$ for some $a \in \mathbb{R}$, such that

$$
-\frac{\int \phi d \mu_{\alpha}}{\lambda\left(\mu_{\alpha}\right)}=\alpha
$$

Proof. As $\int \phi d \mu / \lambda(\mu)$ is a continuous function of $\mu$ (in the weak* topology), we only need to check that there exist such measures $\mu_{\alpha}$ for $\alpha$ arbitrarily close to $\alpha_{\min }$ or $\alpha_{\max }$.

Let us first describe $b(a)$. We will let

$$
s=\sup \{t: P(t \psi)=0\} \text {. }
$$

If $T$ has parabolic points, $P(a \psi) \geq 0$ for any $a \in \mathbb{R}$ and so $b(a)$ is never negative. For $a \leq s$ we have that $b(a)=0$, otherwise it is strictly positive. On the other hand, $b(a)$ can be arbitrarily big since

$$
b(a) \geq \frac{P(a \psi)}{-\inf _{\underline{i}} \phi(\underline{i})}
$$

and so $b(a) \rightarrow \infty$ as $a \rightarrow \infty$. If $T$ does not have any parabolic points, $b(a)$ ranges from $-\infty$ to $\infty$.

Let $\mu_{a}$ be an equilibrium state for $\psi_{a}$ and $\mu$ be any invariant measure. Denote

$$
\alpha=-\frac{\int \phi d \mu}{\lambda(\mu)}
$$

and

$$
\alpha_{a}=-\frac{\int \phi d \mu_{a}}{\lambda\left(\mu_{a}\right)} .
$$

By the variational principle we have

$$
0=h\left(\mu_{a}, \sigma\right)+\int \psi_{a} d \mu_{a} \geq h(\mu, \sigma)+\int \psi_{a} d \mu .
$$

Dividing by $\lambda\left(\mu_{a}\right)$ or by $\lambda(\mu)$ and subtracting, we get

$$
0 \geq b(a)\left(\alpha_{a}-\alpha\right)+\frac{h(\mu, \sigma)}{\lambda(\mu)}-\frac{h\left(\mu_{a}, \sigma\right)}{\lambda\left(\mu_{a}\right)} .
$$

The last summand on the right hand side is bounded by 1 , the second summand is positive. Hence,

$$
\alpha_{a} \leq \alpha+\frac{1}{b(a)}
$$


As $\alpha$ can be chosen to be arbitrarily close to $\alpha_{\min }$ (by the proper choice of $\mu$ ) and $b(a)$ can be chosen to be arbitrarily big (by the proper choice of $a$ ), this means that the corresponding $\alpha_{a}$ can also be chosen arbitrarily close to $\alpha_{\min }$. Similar argument works for $\alpha_{\max }$ if $\alpha_{\max }<\infty$.

If $\alpha_{\max }=\infty$, for $a$ small enough $b(a)=0$ and the Dirac measure at a parabolic orbit is the equilibrium state for $\psi_{a}$. Hence, among the equilibrium states for the family $\left\{\psi_{a}\right\}$ there will be some arbitrarily close (in the weak ${ }^{*}$ topology) to this Dirac measure. For them $\int \psi$ is arbitrarily small while $-\int \phi$ is bounded away from zero. Hence, the ratio $-\int \phi / \int \psi$ is arbitrarily big.

We proved point 1) of Theorem 3 , stating that $\operatorname{dim}_{H} X_{\alpha}$ is a LegendreFenchel transformation of the (sometimes multivalued) function $b^{-1}(a)$. The concavity of $\operatorname{dim}_{H} X_{\alpha}$ follows by standard properties of the LegendreFenchel transformation.

\section{Proof of Theorem 4}

In this section we assume the existence of parabolic periodic orbits. To prove Theorem 4 we will construct an induced system (a hyperbolic expanding map with infinitely many inverse branches) and apply results known for such systems. We construct the induced countable state system, $\bar{T}: \bar{X} \rightarrow \bar{X}$ for our parabolic system as in [7]. We define the functions $\bar{\phi}, \bar{\psi}$ as the induced potentials relating to $\psi$ and $\phi$. For $\alpha \in\left[\alpha_{\min }, \infty\right)$ we define the set

$$
\bar{X}_{\alpha}=\left\{x \in \bar{X}: \lim _{n \rightarrow \infty} \frac{\sum_{i=0}^{n-1} \bar{\phi}\left(T^{i} x\right)}{\sum_{i=0}^{n-1} \bar{\psi}\left(T^{i} x\right)}=\alpha\right\} .
$$

It is immediately clear that

$$
X_{\alpha} \subset \bar{X}_{\alpha} \subset \bigcup_{\beta \leq \alpha} \Pi Y_{\beta}
$$

By (4.6) and monotonicity of $\operatorname{dim}_{H} X_{\alpha}$, it immediately follows that $\operatorname{dim}_{H} X_{\alpha}=\operatorname{dim}_{H} \bar{X}_{\alpha}$. In the case where there exists no SRB measure it is shown in Theorem 7.4 of [7] that $\operatorname{dim}_{H} \bar{X}_{\alpha}$ varies analytically with $\alpha$. In the case where the SRB measure $\mu$ exists, Theorem 7.4 of [7] shows that $\operatorname{dim}_{H} \bar{X}_{\alpha}$ varies analytically with $\alpha$ when $\alpha<-\frac{\int \phi \mathrm{d} \mu}{\int \psi \mathrm{d} \mu}$. For $\alpha$ above this value the function $\alpha \rightarrow \operatorname{dim}_{H} X_{\alpha}$ is constant and equal to $\operatorname{dim}_{H} \Lambda$.

\section{REFERENCES}

[1] L. Barreira, B. Saussol, Variational principles and mixed multifractal spectra, Trans. Amer. Math. Soc. 353 (2001), 3919-3944. 
[2] W. Byrne, Multifractal Analysis of Parabolic Rational Maps, Phd Thesis, The University of North Texas.

[3] R. Cawley and D. Mauldin, Multifractal decompositions of Moran fractals, Adv. Math. 92 (1992), no. 2, 196-236.

[4] G. Contreras, A. O. Lopes, and Ph. Thieullen, Lyapunov minimizing measures for expanding maps of the circle, Ergodic Theory Dynam. Systems 21 (2001), 1379-1409.

[5] K. Gelfert and M. Rams, Geometry of limit set for expansive Markov systems, Trans. Amer. Math. Soc. 361 (2009), 2001-2020.

[6] K. Gelfert and M. Rams, Multifractal analysis of Lyapunov exponents of parabolic iterated function systems, Ergodic Theory Dynam. Systems 29 (2009), 919-940.

[7] P. Hanus, R. Mauldin and M. Urbański, Thermodynamic formalism and multifractal analysis of conformal infinite iterated function systems, Acta Math. Hungar. 96 (2002), 27-98.

[8] F. Hofbauer and P. Raith, The Hausdorff dimension of an ergodic invariant measure for a piecewise monotonic map of the interval, Canad. Math. Bull. 35(1992), no. 1, 84-98.

[9] A. Johansson, T. Jordan, A. Öberg, M. Pollicott, Multifractal analysis of nonuniformly hyperbolic systems, to appear in The Israel Journal of Mathematics, preprint available at www.maths.bris.ac.uk/ matmj/atam103.ps, 2008.

[10] M. Kesseböhmer, Large deviation for weak Gibbs measures and multifractal spectra, Nonlinearity 14 (2001), 395-409.

[11] M. Kesseböhmer and B. Stratmann. A multifractal formalism for growth rates and applications to geometrically finite Kleinian groups, Ergodic theory and dynamical systems 24 (2004), 141-170.

[12] M.Kesseböhmer and B. Stratmann, A multifractal analysis for Stern-Brocot intervals, continued fractions and Diophantine growth rates., J. Reine Angew. Math. 605 (2007), 133-163.

[13] F. Ledrappier, Some properties of absolutely continuous invariant measures on an interval, Ergodic Theory Dynam, Systems 1 (1981), 77-93.

[14] K. Nakaishi, Multifractal formalism for some parabolic maps, Ergodic theory and dynamical systems, 20 (2000), 843-857.

[15] L. Olsen, Multifractal analysis of divergence points of deformed measure theoretical Birkhoff averages, J. Math. Pures Appl. 82 (2003), 1591-1649.

[16] Y. Pesin, Dimension Theory in Dynamical Systems, Contemporary Views and Applications, Chicago Lectures in Mathematics. University of Chicago Press, Chicago 1997.

[17] Y. Pesin and H. Weiss, The multifractal analysis of Birkhoff averages and large deviations, Global analysis of dynamical systems, 419-431, Inst. Phys., Bristol, 2001.

[18] D. Rand, The singularity spectrum $f(\alpha)$ for cookie-cutters, Ergodic Theory Dynam. Systems 9 (1989), no. 3, 527-541.

[19] B. Stratmann and M. Urbański, Real Analyticity of Topological Pressure for parabolically semihyperbolic generalized polynomial-like maps, Indag. Mathem. 14 (2003), 119-134. 
[20] B. Stratmann and M. Urbański, Multifractal analysis for parabolically semihyperbolic generalized polynomial-like maps, IP New Studies in Advanced Mathematics, 5 (2004), 393-347.

[21] M. Urbański, Parabolic Cantor sets Fund. Math. 151 (1996), 241-277.

[22] P. Walters, An Introduction to Ergodic Theory, Springer, 1982.

[23] M. Yuri, Weak Gibbs measures for certain non-hyperbolic systems, Ergodic Theory Dynam. Systems 20 (2000), 1495-1518.

[24] M. Yuri, Multifractal analysis of weak Gibbs measures for intermittent systems, Comm. Math. Phys. 230 (2002), no. 2, 365-388.

Thomas Jordan, Department of Mathematics, The University of Bristol, University Walk, Clifton, Bristol, BS8 1TW, UK

E-mail address: thomas.jordan@bristol.ac.uk

Michą Rams, Institute of Mathematics, Polish Academy of SciENCES, Ul. ŚNIADECKich 8, 00-956 WarszaWa, Poland

E-mail address: M.Rams@impan.gov.pl 